\section{Ticagrelor not superior to DAPT after $\mathrm{PCl}$}

Although dual antiplatelet therapy (DAPT) remains the standard treatment to prevent stent thrombosis after percutaneous coronary intervention (PCI), ongoing trials are investigating alternative strategies such as a shorter duration of DAPT followed by monotherapy to reduce the risk of bleeding. According to data from the randomized GLOBAL LEADERS trial presented at the 2018 ESC Congress, treatment with ticagrelor for 2 years after PCI (with aspirin during the first month) shows no benefit compared with standard DAPT.

The initial hypothesis was that ticagrelor, a potent $\mathrm{P} 2 \mathrm{Y}_{12}$-receptor antagonist that reduces adverse cardiac outcomes in patients with acute coronary syndrome (ACS), could prevent ischaemic events while avoiding bleeding complications in patients after PCI.

A total of 15,968 patients with stable coronary artery disease (CAD) or ACS undergoing PCI with drug-eluting stent implantation were randomly assigned to receive aspirin and ticagrelor daily for 1 month, followed by 23 months of ticagrelor monotherapy, or standard DAPT with aspirin and another antiplatelet agent - either clopidogrel for stable CAD or ticagrelor for ACS - daily for 1 year, followed by aspirin monotherapy for 1 year.

At 2 years, the primary end point of all-cause mortality or nonfatal new Q-wave myocardial infarction occurred in $3.81 \%$ of participants in the experimental group and $4.37 \%$ of participants in the control group (rate ratio $0.87,95 \% \mathrm{CI}$ $0.75-1.01, P=0.073$ ), and the frequency of bleeding was similar between the groups. Subgroup analysis also revealed no significant difference in the effects of the treatments in patients with stable CAD versus those with ACS.

In conclusion, while other trials on antiplatelet strategies are underway, the results of this trial do not support a change in clinical practice at this time.

Alexandra Le Bras

ORIGINAL ARTICLE Vranckx, P. et al. Ticagrelor plus aspirin for 1 month, followed by ticagrelor monotherapy for 23 months vs aspirin plus clopidogrel or ticagrelor for 12 months, followed by aspirin monotherapy for 12 months after implantation of a drug-eluting stent: a multicentre, openlabel, randomised superiority trial. Lancet 392, 940-949 (2018) FURTHER READING Miyazaki, Y. et al. Single or dual antiplatelet therapy after PCI. Nat. Rev. Cardiol. 14, 294-303 (2017)

\title{
No benefit of MitraClip for secondary mitral regurgitation in heart failure
}

The presence of secondary mitral regurgitation, characterized by a structurally normal valve with regurgitation resulting from a dilated left ventricle with poor function, is a predictor of poor clinical outcomes for patients with heart failure. Reducing the regurgitation with surgery or percutaneous intervention has been proposed to improve outcomes in these patients; in particular, percutaneous repair with a MitraClip (Abbott Vascular) is becoming more frequent. However, only low-level evidence is available to support these approaches. A randomized clinical trial presented at the ESC Congress now shows that in patients with chronic heart failure and severe secondary mitral regurgitation, percutaneous mitral valve repair with a MitraClip in addition to medical therapy, although effective in reducing regurgitation, does not improve survival or symptoms compared with medical therapy alone.

The trial included 304 patients with severe secondary mitral regurgitation and symptomatic heart failure with left ventricular ejection fraction $15-40 \%$ who were randomly assigned to percutaneous mitral valve repair with a MitraClip in addition to optimal medical treatment or to optimal medical therapy alone. The MitraClip was safe and significantly reduced mitral regurgitation, with a procedural success rate of $95.8 \%$. However, after 1 year, the rate of the primary end point (all-cause death or unplanned hospitalization for heart failure) and the rates of the individual components were similar in both groups.

"These results should encourage a more accurate selection of patients with heart failure before considering mitral valve repair," remarks lead investigator Jean-François Obadia. "More randomized studies are necessary to define possible subgroups of patients who could really benefit from mitral valve repair," he concludes.

\section{Irene Fernández-Ruiz}

ORIGINAL ARTICLE Obadia, J.-F. et al. Percutaneous repair or medical treatment for secondary mitral regurgitation. N. Engl.J.Med. https://doi.org/10.1056/NEJMoa1805374 (2018)
OBESITY

Cardiovascular safety of lorcaserin

The weight-loss drug lorcaserin is safe and not associated with an increased rate of cardiovascular events, according to data presented at the 2018 ESC Congress. Lorcaserin is a selective agonist of the 5-hydroxytryptamine receptor $2 \mathrm{C}$, which is involved in the regulation of appetite. The drug was shown in previous trials to be effective for promoting weight loss and was consequently approved by the FDA in 2012 as an adjunct to a reduced-calorie diet and increased physical activity for longterm weight management. However, other weight-loss drugs have had an adverse cardiovascular risk profile, so the CAMELLIA-TIMI 61 trial was initiated to test the cardiovascular safety and efficacy of lorcaserin.

A total of 12,000 patients who were overweight or obese (BMI $\geq 27)$ with established atherosclerotic cardiovascular disease or multiple cardiovascular risk factors were randomly assigned to receive either lorcaserin (10 mg twice daily) or placebo. At 1 year, weight loss of $\geq 5 \%$ had occurred in $38.7 \%$ of patients in the lorcaserin group and in $17.4 \%$ of patients in the placebo group (OR 3.01, 95\% Cl 2.74-3.30, $P<0.001$ ).

During follow-up (median 3.3 years), the rate of the primary safety outcome (a composite of cardiovascular death, myocardial infarction, or stroke) was $2.0 \%$ and $2.1 \%$ per year in each group, respectively (HR 0.99, 95\% Cl 0.85-1.14, $P<0.001$ for noninferiority). The rate of the primary cardiovascular efficacy end point (extended major cardiovascular events) was not significantly different between the two groups ( $4.1 \%$ versus $4.2 \%$ per year).

The rates of adverse events were generally similar in both groups; however, more patients in the lorcaserin group than in the placebo group developed serious hypoglycaemia (13 versus 4; $P=0.04$ ). "Liraglutide [a GLP1 receptor agonist] would provide a similar degree of weight loss but a lower risk of diabetes [mellitus]," comment Julie Ingelfinger and Clifford Rosen in an accompanying editorial. Accordingly, they advise that lorcaserin "may be best used on a cautious basis according to the needs of individual patients".

Gregory B. Lim

ORIGINAL ARTICLE Bohula, E. A. et al. Cardiovascular safety of lorcaserin in overweight or obese patients. N. Engl.J. Med. https://doi.org/10.1056/NEJMoa1808721 (2018) 